International Journal of Engineering \& Technology, $7(1)(2018) 85-89$
SPC
International Journal of Engineering \& Technology
Website: www.sciencepubco.com/index.php/IJET
doi: $10.14419 /$ ijet. $v 7$ il. 8330
Research paper

\title{
An analysis of springback of compliant assemblies by contact modeling and welding distortion
}

\author{
Atik Hafsa*, Mouhssine Chahbouni, Boutahari Said, Amegouz Driss \\ High School of Technology of Fez, University sidi mohamed ben abdellah B.P 2626 -Route d'Imouzzar,30000 Fez,Morocco. \\ *Corresponding author E-mail:hafsa.atik@usmba.ac.ma
}

\begin{abstract}
Due to the development of the automotive and aeronautical industries, and the impossibility of pro-typing of the flexible parts because of the large real dimension as well as the behavior of this type of parts during the assembly, the tolerance of the flexible parts become an essential step in aeronautical manufacturing. Therefore the tolerance of plates in the assembly of mechanical systems is one of the key stages in the creation of a product in the automotive and aeronautical industries. This paper deals in the first stage a presentation of the tolerance of deformable mechanisms, through the illustration of the general problem. In order to study in the second stage the model of simulation of the variation of deformable (flexible) mechanisms, using the Influence Coefficient Method taking into account the effects of contact between the surface and including welding distortion. Finally the modeling of a mechanism of this type through an example with a view to an analysis of tolerances.
\end{abstract}

Keywords: Tolerance Analysis, Deformable Mechanisms, Influence Coefficients Method, Surface Contact, Welding Distortion.

\section{Introduction}

Sheet metal assembly is broadly used in industries. Because of the complexity of the fabrication, and the assembly of this type of part, generally they are assembled by deformation during assembly. Part and process variation will influence the final geometry and variation of the assembly. Tolerance analysis is a way to predict the consequences of the geometrical defects on the assembly to be avoided during early design stages. The frequently used models are the Influence Coefficient Method (MIC) [1] [2], Monte Carlo method (MMC), [2] [3] [4] and Finite Element Methods (FEM) [5]. Researchers have proposed different tolerance analysis methods. Liu and $\mathrm{Hu}[1]$ used the finite elements method to obtain a relation between the initial tolerances and the final deformation of the assembly by assuming a linear force-displacement relation and calculating the related sensitivity matrix, this method is named Influence Coefficient Method (MIC). The effect of Contact interaction between assembled surfaces it was not considered, this disadvantage of the original method allows the parts to penetrate each other which influences the final results thus being far from the real modeling of the assembly this is indeed the target of several works later. Stefan Dahlström and Lars [6] [7] propose a contact algorithm that will be implemented into the method. As well as Khodaygan [8] who attribute the same problem. For more performance, Wooyoung [9] proposes a model that extends the concepts of the sources of variation and the Influence Coefficient Method for a compliant mechanical assembly to include the welding distortions. Additionally, the work of Alain [3] [10], that are based principally on the development of the Influence Coefficient Method, but this time with taking into account the variation of rigidity induced by the defaults of the form and finally the applica- tion on a large structure of the aerospace industry and finally we have important works in this subject [11] [12].

For the same consideration, and in the same direction of performance, and improvement of modeling and analysis of flexible assembly. The present work proposes the simulation of the deformable (flexible) mechanisms using the Influence Coefficient Method taking into account the shape defects of each part, including the effects of the contact between surface and welding distortion. The performances of this proposed method compared with a simulation with contact and without contact using the Influence Coefficient Method in term of accuracy and computation cost.

\section{Influence Coefficient Method}

The principle of the Influence Coefficient Method is to establish a linear model between part deviations and assembly springback deviations, and that's why it is a way of performing the simulation faster than direct Monte Carlo. This method consists of three steps:

1. Force response on the displacements. A unit force is applied to the $\mathrm{j}$-th source of variation $(\mathrm{j}=\mathrm{I}$ to $\mathrm{N}$ ) on the part. The source of variation could be any type of variation that the part will be subjected to. The response under that unit force the deformation of the part is calculated by Finite Element Analysis (FEA), and the displacements $(\mathrm{i}=1$ to $\mathrm{M})$ of the parts are recorded into a column vector $\mathrm{Cj}$. We consider that the system is applied by an arbitrary combination of $\mathrm{N}$ forces $(\mathrm{Fj}, \mathrm{j}=1$ to $\mathrm{N})$. [C] Is the matrix of influence coefficients, which the vector $\{\mathrm{V}\}$ represents the deviations at the sources of variation. 


$$
\{\mathrm{V}\}=[\mathrm{C}]\{\mathrm{F}\}
$$

2. Force response on the fixture points. In step one when a unit force is applied to the $\mathrm{j}$-th source of variation, the reaction forces of the fixture points are recorded into a column vector $\mathrm{Dj}$. The reaction forces $\mathrm{R}$ are the forces released during the springback process and [f] is the applied forces.

$$
\{R\}=[D]\{f\}
$$

3. Springback calculation of the assembly. The springback displacements for the assembly are calculated with FEA similarly to step 1. The displacements of the points of interest are recorded into a column vector $\mathrm{Sj}$ for the assembly or the join parts. Here, the forces $\mathrm{F}$ are applied only to the fixture points or clamps that are released when the joining is done. Linear models between the applied forces $\mathrm{F}$ on the released fixture points and the springback displacements on final assembly could be derived by combining all the vectors $S j$ into a matrix:

$$
\{\mathrm{U}\}=[\mathrm{S}]\{\mathrm{V}\}
$$

\section{Contact Modeling}

Consider a problem of three-dimensional contact with friction between two deformable bodies Q1 target (Target) and Q2 contactor (representative), shown in Fig. 2. In Q1 and Q2, the limits Ld and Ls are the displacements applied and the forces / moments, respectively. The contact surfaces are designated Lc (1) and Lc (2), respectively. Defining a common contact surface Lc, located between Lc (1) and Lc (2), and an orthonormal local reference surface $(\mathrm{t} 1, \mathrm{t} 2, \mathrm{n})$ in each mode, where $(\mathrm{t} 1, \mathrm{t} 2)$ represents the plane tangent to the surface and $n$ is the Outside the surface.

$(\mathrm{t} 1, \mathrm{t} 2)$ represents the plane tangent to the surface and $\mathrm{n}$ is the normal to the surface. Suppose that the displacement vector and the contact force vectors in the orthonormal local reference are: pc $=(\mathrm{pt} 1, \mathrm{pt} 2, \mathrm{pn})$, respectively. Here pn indicates the normal contact force and the contact friction force pt is decomposed into components $\mathrm{pt} 1$ and $\mathrm{pt} 2$.In order to prevent the bodies from penetrating one another, the normal contact distance (gn: $=\mathrm{u} 1-\mathrm{u} 2-\mathrm{d} 0$ ) where $\mathrm{d} 0$ is the initial deviation on the contact boundary Lc must be greater than or equal to Zero, i.e. gn $\geq 0$. The contact between the two bodies Q1 and Q2 shown in the Figure is now locally characterized by a one-sided contact condition, condition compression, and a complementary condition as follows:

Unilateral contact condition: gn $\geq 0$,

Compression condition: $\mathrm{pn} \leq 0$,

Supplementary condition: $\mathrm{pn} \cdot \mathrm{gn}=0$,

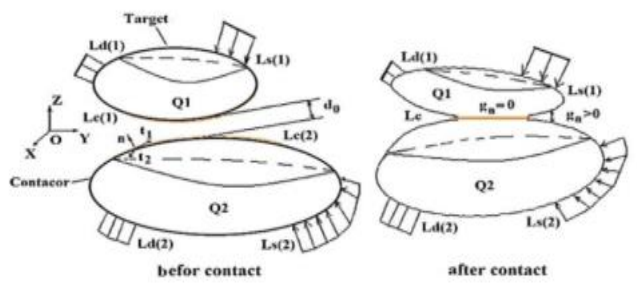

Fig1: General model of the elastic contact problem

\section{Welding Distortion}

A limitation of the Influence Coefficient Method is the taking into account welding distortion. Considering sheet metal assembly process typically consists of the following steps: loading sheet metal parts to work-holding fixtures; clamping parts to nominal positions; welding the sheet metal parts and releasing the clamps and /or fixtures [1].When we neglect welding deformation we can write:

$\left\{\mathrm{F}_{\mathrm{w}}\right\}=\left[\mathrm{K}_{\mathrm{w}}\right]\left\{\mathrm{U}_{\mathrm{w}}\right\}=\left[\mathrm{k}_{\mathrm{u}}\right]\left\{\mathrm{V}_{\mathrm{u}}\right\}=\left\{\mathrm{F}_{\mathrm{u}}\right\}$

$\left\{\mathrm{U}_{\mathrm{w}}\right\}=\left[\mathrm{K}_{\mathrm{w}}\right]^{-1}\left[\mathrm{~K}_{\mathrm{u}}\right]\left\{\mathrm{V}_{\mathrm{u}}\right\}=\left[\mathrm{S}_{\mathrm{u}}\right]\left\{\mathrm{V}_{\mathrm{u}}\right\}$

With $\left\{\mathrm{U}_{\mathrm{w}}\right\}=\left[\mathrm{K}_{\mathrm{w}}\right]^{-1}\left\{\mathrm{~F}_{\mathrm{w}}\right\}$

$\left\{F_{\mathrm{u}}\right\}$ The clamping force of the unwelded Structure

$\left\{\mathrm{F}_{\mathrm{w}}\right\}$ The clamping force of the welded structure

$\left[\mathrm{K}_{\mathrm{u}}\right]$ The stiffness matrix of the unwelded structures

$\left[\mathrm{K}_{\mathrm{W}}\right]$ The stiffness matrix of the welded structures

$\left\{\mathrm{V}_{\mathrm{u}}\right\}$ The part variation

$\left\{\mathrm{U}_{\mathrm{w}}\right\}$ The assembly deviation

$\left[\mathrm{S}_{\mathrm{u}}\right]$ The sensitivity matrix

To consider the effects of welding distortion, we included welding distortion as a form of the equivalent force in the MIC. It assumes that the distortion generated during the welding of non-nominal parts is identical to that of nominal parts. Also, it assumes all the welding distortions are deterministic. The following equations describe the details of the MMIC:

$\left[\mathrm{K}_{\mathrm{w}}\right]\left\{\mathrm{U}_{\mathrm{w}}\right\}-\left\{\mathrm{F}_{\mathrm{d}}\right\}=\left[\mathrm{S}_{\mathrm{u}}\right]\left\{\mathrm{V}_{\mathrm{u}}\right\}$

$\left\{\mathrm{U}_{\mathrm{w}}\right\}=\left[\mathrm{S}_{\mathrm{u}}\right]\left\{\mathrm{V}_{\mathrm{u}}\right\}+\left[\mathrm{K}_{\mathrm{w}}\right]^{-1}\left[\mathrm{~F}_{\mathrm{d}}\right]$

$\left\{\mathrm{U}_{\mathrm{w}}\right\}=\left[\mathrm{S}_{\mathrm{u}}\right]\left\{\mathrm{V}_{\mathrm{u}}\right\}+\{\mathrm{D}\}$

In this way we can expressed the assembly deviation and we have added the displacement vector due to welding $\{D\} .\left\{F_{d}\right\}$ represents the additional clamping force caused by welding distortion and $\left[\mathrm{K}_{\mathrm{W}}\right]^{-1}\left[\mathrm{~F}_{\mathrm{d}}\right]$ is interpreted as the equivalent force to the welding distortion.

\section{Proposed Method}

The procedure for MIC including the effects of contact between the surface and welding distortion is the same as for ordinary MIC, with the exception that sensitivity matrices the proposed method entails the following workflow in Figure 2 who showed dimensional variation analysis by contact modeling, and welding distortion for assembly follows this step:

- Apply variation to the parts.

- Apply welding and clamping variation.

- Calculation of reaction forces and welding distortion.

- Detection of the contact between parts

- Verification of the condition

- Verification if the reaction forces R and the final distances d:

$$
\begin{gathered}
\text { All: } \mathrm{d}_{\mathrm{i}} \geq 0 \\
\text { All: } \mathrm{R}_{\mathrm{i}} \leq 0 \\
\text { All: } \mathrm{d}_{\mathrm{i}} \mathrm{R}_{\mathrm{i}}=0
\end{gathered}
$$

- Calculation of the final displacement 


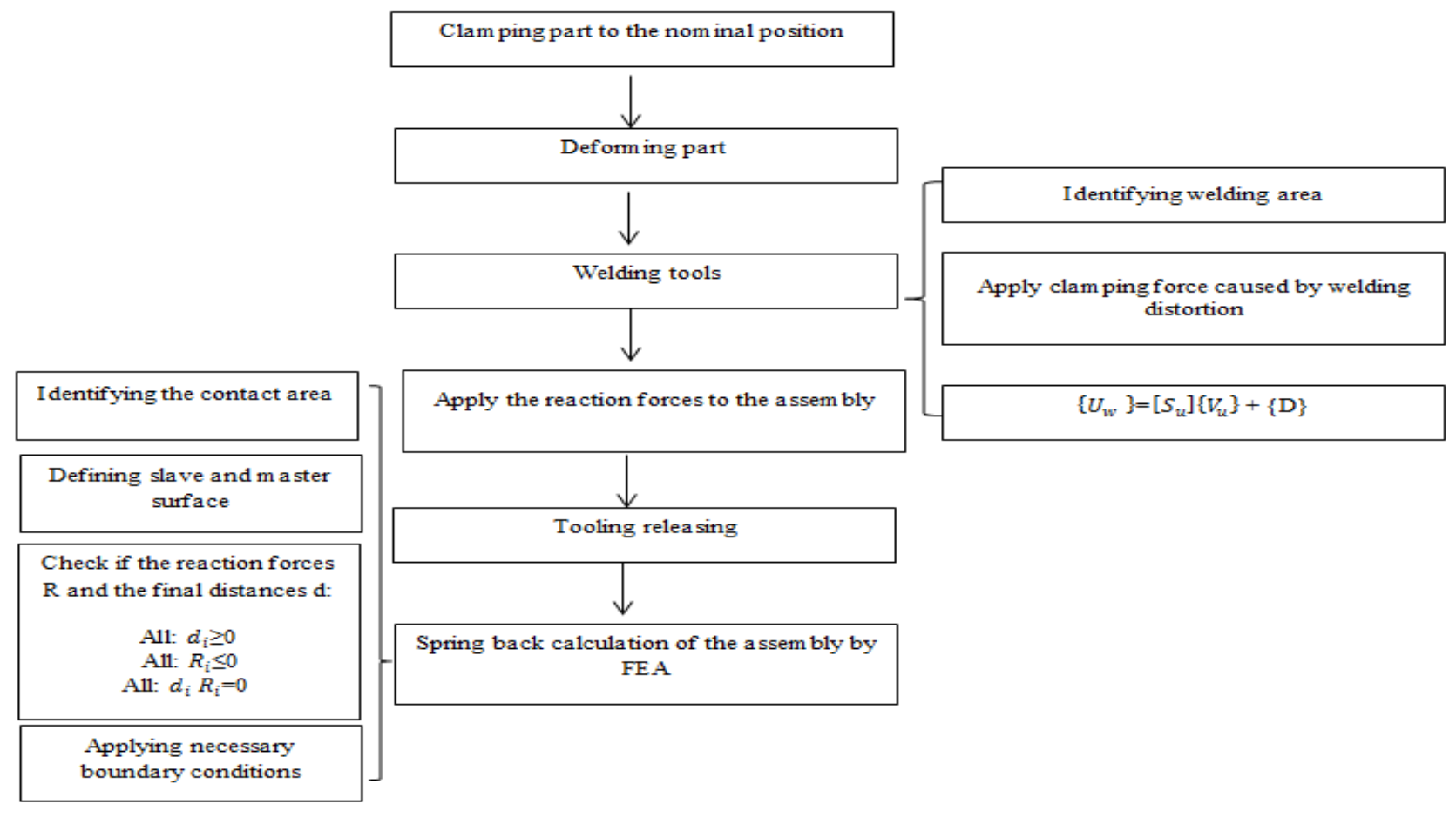

Fig2: The workflow for assembly dimensional variation analysis by contact modeling, and welding distortion

\section{Case Study 1}

For validation purposes, we compare simulation data of MIC including contact modeling with the variation simulation results including the welding distortion and contact modeling, these case studies propose to perform tolerance analysis on compliant assemblies. We use a two plate assembly as an example to illustrate the variation simulation of the deformable (flexible) assembly using the Influence Coefficient Method included the effect of contact modeling and to limit shape defects and connection defects and take into consideration the welding distortions during the joining process, to guarantee a simulation closest to the real. This case study explains how the initial part variations are propagated to the welded assembly, including the variation from the welding distortion.

The aim of the example: is to analysis the thin plate by the Influence Coefficient Method with consideration contact modeling and welding distortions.

Problem: To estimate the behavior of compliant assemblies with a method to take into account the variation of each part under the effect of an impulsive load. It is often necessary to know the final state of the structure. This state allows the designer to dimension the plate. The example used is an assembly of two pieces of sheet metal joined by welding as shown in figure 3 .We are interested in finding the mean deviation of the final assemble.

\subsection{Hypothesis}

The two plates are assumed to have some initial variation. The two plates are considered with form deviation. Functional condition: final deformation of the assembly enters the tolerance zone.

\subsection{Geometry and properties of materials}

The dimension of this plate is $700 \mathrm{~mm} \times 300 \mathrm{~mm} \times 1 \mathrm{~mm}$ and the same for the second. and The material of both parts is soft steel with a Young's modulus $\mathrm{E}=20,700 \mathrm{~N} / \mathrm{mm}^{2}$ and poison's coefficient is $\mathrm{v}=0,3$.

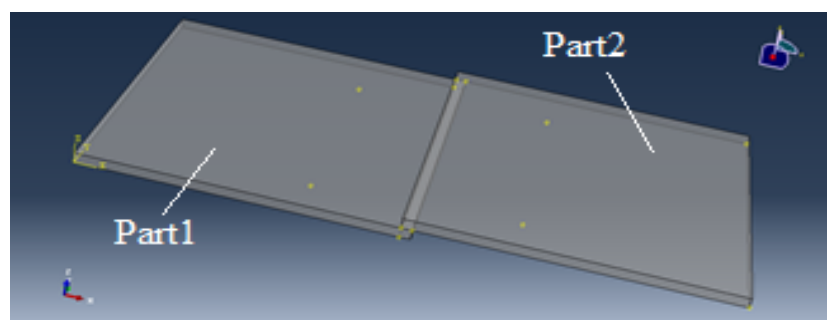

Fig3: Geometrical model of the plate assembly

Sources of variation and boundaries conditions

In the first step of Influence Coefficient Method we need to apply each source of variation for each part (here we have $\mathrm{V} 1, \mathrm{~V} 3$ are the sources of variation for the part one and V2,V4 are the sources of variation for part two) to calculate sensitivity matrix and reaction forces in this step the parts are not joined.

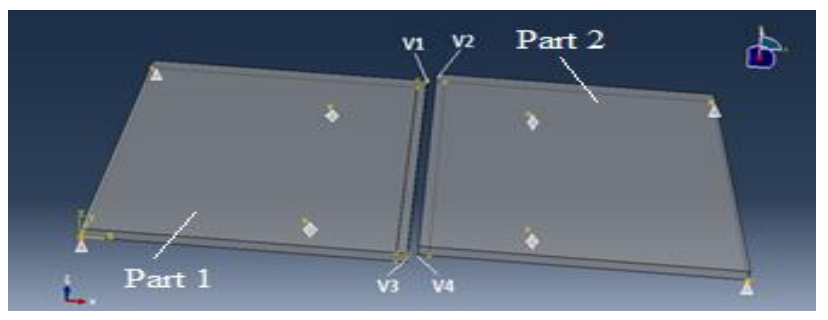

Fig4: Sources of variations and boundary conditions of the parts $\vartheta, \triangle \quad \begin{aligned} & \text { Boundary condition } \\ & \text { Source of variation }\end{aligned}$

Here, the parts of the assembly are joined and we apply reaction forces R1, R2, R3, R4, R5, R6, R7, R8 calculated by MIC 
method with contact Modelling and Source of variation, the detailed welding scheme is shown in Figure 5 where the red line represents a weld and the contact area between the parts is defined. Finally we can calculate the springback of the assembly. And then we do the same thing, but this time with method of influence coefficient with contact modelling and effect of welding

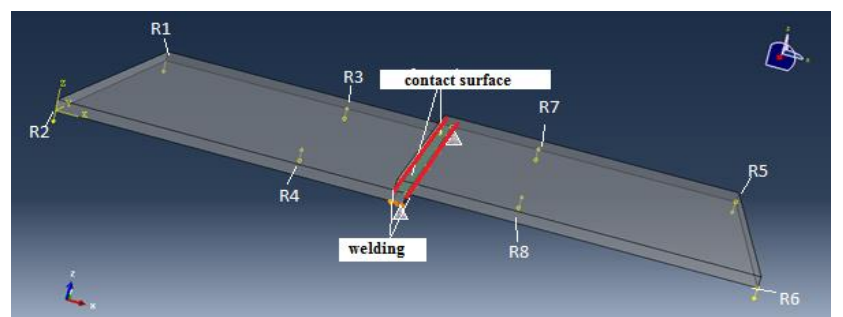

Fig5: Reaction force and boundary condition for the welded sheet metal assembly

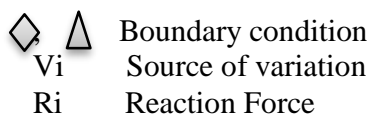

\subsection{Simulation results}

In order to verify and test the implemented contact algorithm in MIC, two different simulations for the same geometry and variation are performed:

-Influence Coefficient Method with contact modelling -Influence Coefficient Method with contact modelling and effect of welding.

The final displacement for all nodes after the springback deflection is recorded for every iteration. The same set of variables has been used in all simulations.

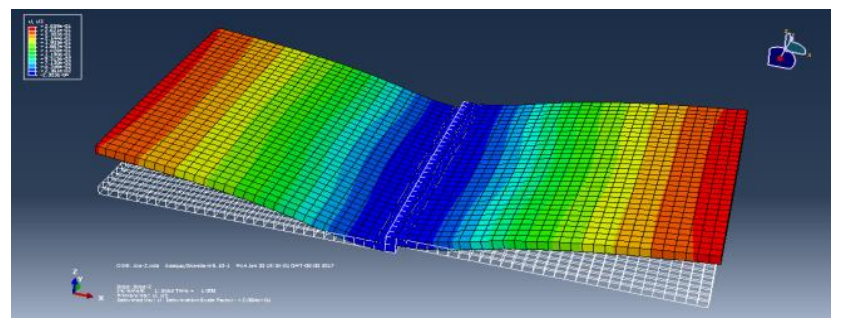

Fig6: Simulation result of proposed model

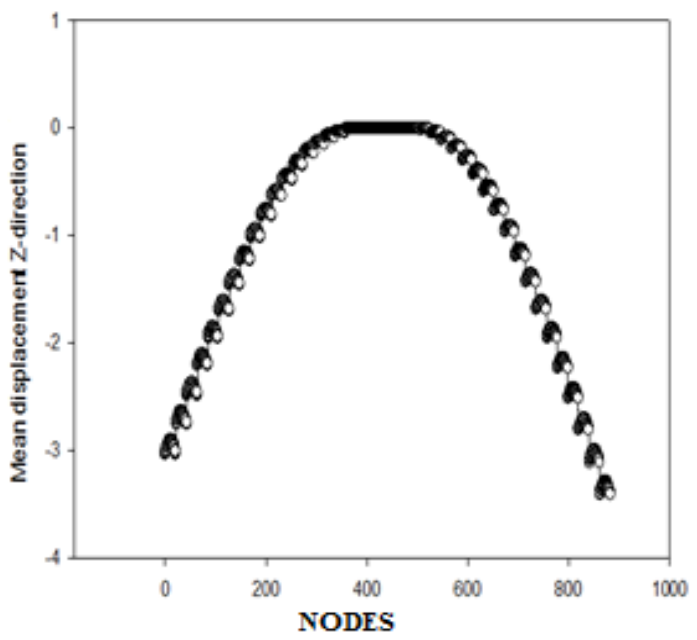

- Nodes vs Uethad of Coefficients Iffuence weth cortact modeling and efect d weling - Nodes vs llethod of Coefficients Iffuence weth contact modeling

Fig7: Comparison method of influence coefficient with contact modelling and with contact modelling and effect of welding

\subsection{Comparison results and discussion}

This comparison shows that, when contact modeling and welding distortion are not used, the result from MIC is not the same as the simulation of MIC with contact modeling and effect of welding. This means that MIC when contact modeling and welding distortion is more precise than MIC classic because the modelization of the mechanism it more near to the real mechanism. And the MIC classic allows the penetration between the plates unlike MIC with contact modelling and effect of welding.

\section{Case study 2}

In this second case with the same aim and the same problem we are studying an assembly for a cross tube and a differential mounting bracket who was welded as shown in figure 8 where the red line represents a weld and we have also a contact between cross tube and a differential mounting bracket. After application of method of influence coefficient with contact modelling and with contact modelling and effect of welding we calculate reaction forces R1, R2, and R3 we calculated and recorded displacement after springback of the assembly as shown in figure 8.

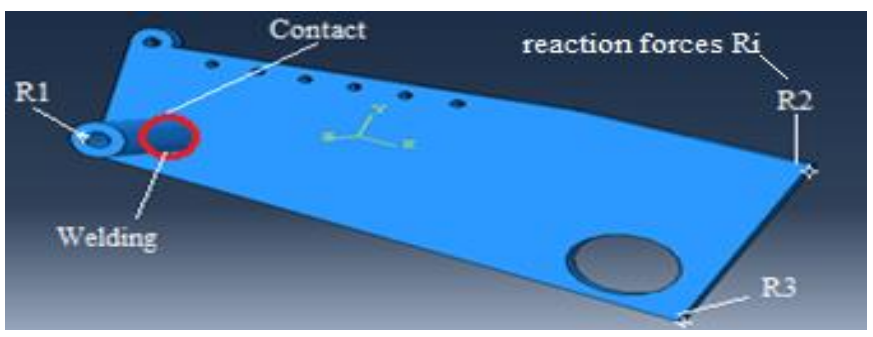

Fig8: The cross tube was welded to the differential mounting bracket

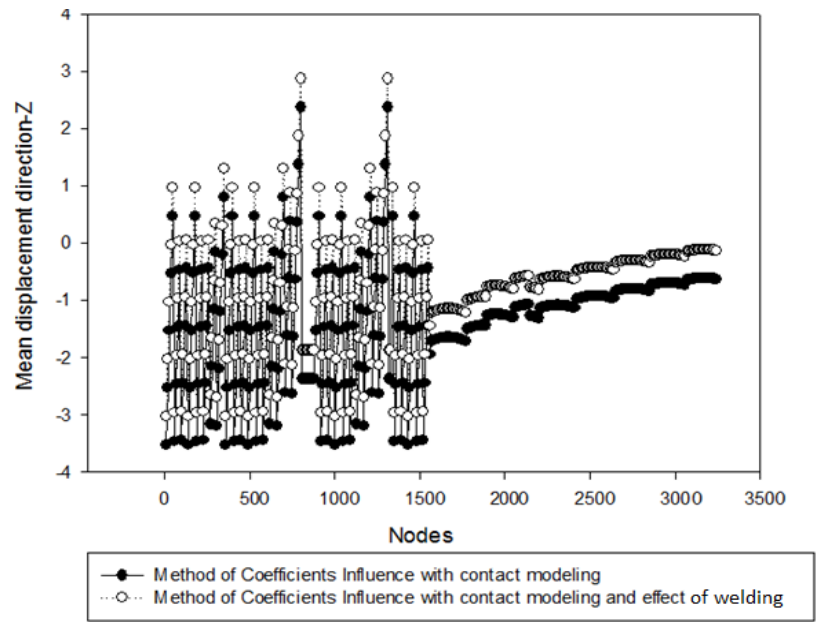

Fig9: Comparison method of influence coefficient with contact modelling and with contact modelling and effect of welding

The second comparison shows that, in this case and probably many others, the result without contact modeling and welding distortion is completely erroneous. The reason for this is without contact modeling; the flanges are allowed to penetrate each other and without welding distortion can have different deformation in this zone.

\section{Conclusion}

This paper has presented a contact and welding distortion algorithm, which can be implemented into the Influence Coefficient Method, and how these two parameters can influence the assembly process that affects the assembly dimensional quality. 
This algorithm has been verified with two case studies. First one is a simple example of two plates that have been assembled by welding and the second one is an industrial example of an assembly for a cross tube and a differential mounting bracket. The analysis of these case studies shows that variation simulation with Influence Coefficient Method with contact modelling and welding distortion effect can be simulated with good accuracy and precision compared simulation with Influence Coefficients Method with contact modelling. Finally, the capabilities of the proposed method and the accuracy of the results were investigated through a case study of an assembly of industrial example of an assembly for a cross tube and a differential mounting bracket.

\section{References}

[1] Liu, S. C., and Hu, S. J., 1997. "Variation Simulation for Deformable Sheet Metal Assemblies Using Finite Element Methods". Journal of Manufacturing Science and Engineering, 119(3), Aug., pp. 368-374.

[2] S.C. Liu, S.J. Hu, T.C. Woo, Tolerance analysis for sheet metal assemblies, ASME J. Mech. Des. 118 (1) (1996) 62-67.

[3] Stricher, A., Champaney, L., Thiebaut, F., Fricero, B., and Chevassus, N., 2011. "Mod' ele simplifi' e pour la simulation d'assemblage de plaques planes avec d' efauts de forme.". In 12 eme Colloque National AIP PRIMECA, no. 1, pp. 1-9.

[4] Merkley, K. G., 1998. "Tolerance analysis of compliantassemblies". PhD thesis, Brigham Young University.

[5] Xiaoyun , L, Gary Wang,G, "Non-linear dimensional variation analysis for sheet metal assemblies by contact modeling". Finite Elements in Analysis and Design 44 (2007) pp. 34 - 44

[6] Dahlstrom .S, L. Lindkvist, "Contact modeling in method of influencecoefficient for variation simulation of sheet metal assemblies", in: Proceedings of IMECE04, 2004 ASME International Mechanical Engineering Congress and Exposition, Anaheim, CA, USA, November 13-20, 2004 (Paper Number: IMECE2004-61550).

[7] Dahlstrom. S, J.S. Hu, R. Soderberg, "Identifying variable effects on the dimensional quality of compliant assembly using computer experiments", in: Proceedings of DETC'02, ASME 2002 Design Engineering Technical Conferences and Computers and Information in Engineering Conference,Montreal, Canada, September 29-October 2, 2002 (Paper Number:DETC2002/DAC-34035).

[8] Khodaygan .S, "Statistical Tolerance Analysis of Flexible Assemblies with Contact Effects" Conference Paper April 2016, SAE International.

[9] Wooyoung, C, Hyun, C, "Variation Simulation of Compliant Metal Plate Assemblies Considering Welding Distortion" in Journal of Manufacturing Science and Engineering. Febuary 9, 2015 by ASME.

[10] Stricher.A, "Tolérancement flexible d'assemblages de grandes structures aéronautiques," École normale supérieure de Cachan ENS Cachan, 2013.

[11] Mattikalli,.R "Modeling compliant part assembly: mechanics of deformation and contact", in: Proceedings of DETC'00, 2000 ASME Design Engineering Technical Conferences, September 10-13, 2000, Baltimore, MD, USA (Paper Number: DETC2002/DFM-14036).

[12] Chahbouni.M, boutahari.S, "Influence of form deviations on the tolerance analysis" International Journal of Engineering \&Technology 2014 paper 343-349. 